\title{
ELECTRONCONCENTRATION AND SPECTRAL INTENSITY DISTRIBUTION IN A CESIUM DISCHARGE
}

\author{
By Fred L. Mohler
}

ABSTRACT

The theoretical relation between the transition probabilities for line emission in a given series and the probability of recombination into the final state of the series is derived from the postulate that the line intensity approaches the continuous intensity at the limit in thermal equilibrium. The measured values of $A$ for the subordinate series of cesium lead to a value $1.7 \times 10^{-21} \mathrm{~cm}^{2}$ for the effective cross section for recombination of a 0.3 -volt electron into the $6 \mathrm{P}$ limit. Measurements of recombination intensity and electron concentration have led to an identical value at low pressures, but the value apparently increased with pressure. It is concluded that probe measurements are systematically low at higher pressures and the intensity of the continuous spectrum is used as a basis for computing the true values of electron concentration $N_{\text {e. }}$.

It has been shown that at pressures above $60 \mu$ all the higher series lines have the same reversal temperature. One can define a temperature, $T_{s}$, by a given value of $N_{e}$ in Saha's equation and the values so derived are equal to the reversal temperatures of the higher series lines. $T_{s}$ is always less than the electron temperature but the two approach each other with increasing pressure.

\section{CONTENTS}

Page

I. Introduction

II. Relation between the probability of recombination and the line transition probabilities

III. Probability of recombination into $6 \mathrm{P}$ of cesium 851

IV. Electron concentration from intensity measurements.. 852

V. Equilibrium between excited states and ionized states_._.

\section{INTRODUCTION}

A series ${ }^{1}$ of papers has described a systematic study of the columnar discharge in cesium vapor in which various phenomena have been studied as a function of vapor pressure and current in a tube $1.8 \mathrm{~cm}$ in diameter. The data include electrical measurements of electron concentration and temperature, measurements of the number of excited atoms in different excited states, and measurements of intensity distribution in the line spectrum and in the continuous recombination spectrum. The purpose of finding general relations between the intensity distribution and the electrical conditions was not fully realized because of an apparent contradiction in the experimental data. In this paper the contradiction is removed by a more or less arbitrary

1 F. L. Mohler, Power input and dissipation in the positive column of a caesium discharge, BS J. Research 9 , 25 (1932) RP455.

Collision of the first and second kind in the positive column of a caesium discharge, BS J. Research 9, 493 (1932) RP485.

Recombination radiation in the cesium positive column, BS J. Research 10, 771 (1933) RP565.

Reversal temperature and population of excited states in the cesium discharge, J. Research NBS 16, 227 (1936) RP869.

Intensity distribution in the line emission spectrum of cesium, J. Research NBS 17, 45 (1936) RP901. 
choice between conflicting results, and a general relation is thereby derived which seems to justify the choice.

The dilemma arose in the measurements of the intensity of the continuous recombination spectrum as a function of the number of electrons per cubic centimeter, $N_{e}$. One would expect the intensity to depend only on the number of electrons and ions and on the electron velocity distribution. The experiments indicated that the intensity was from 10 to 40 times as great at a pressure of 300 microns of mercury as at 1 micron for equal values of $N_{e}$. This led to the assumption that the probability of recombination with emission of radiation depended on the vapor pressure. ${ }^{2}$ The rate of recombination at higher pressures is known to depend on pressure, but the conventional view is that the process is radiationless. An alternative possibility is that the probe wire measurements of $N_{e}$ are systematically in error at the higher pressures. Studies of the line intensity as a function of discharge conditions and consideration of the theoretical relation between line intensity and continuous intensity support the second alternative. $^{3}$

\section{RELATION BETWEEN THE PROBABILITY OF RECOMBI- NATION AND THE LINE TRANSITION PROBABILITIES}

It is a familiar fact that an intense continuous spectrum beyond a series limit is always accompanied by relatively intense higher series lines which gradually merge into the continuum 50 or $100 \mathrm{~A}$ on the long wave length side of the theoretical limit and there is no perceptible discontinuity at the limit. The relation between line transition probabilities and the probability of recombination can conveniently be derived from the postulate that in thermal equilibrium the line intensity and the continuous intensity must similarly approach equality at the limit.

The intensity in ergs per cubic centimeter of radiation from a transition from the $n$th state of a series into a common final state is

$$
J_{n}=N_{n} A_{n} h \nu,
$$

where $A_{n}$ is the transition probability and $N_{n}$ is the population of the $n$th state. At a temperature, $T$,

$$
N_{n}=N_{1} \frac{g_{n}}{g_{1}} \exp .\left(-E_{n} / k T\right),
$$

where $E_{n}$ is the energy of the $n$th state relative to the normal state, $N_{1}$ is the population of the normal state, and $g_{n}$ and $g_{1}$ are the weights of the two states. The average frequency interval between two successive lines of a hydrogen series, or any series if $n$ is large, is

$$
\delta \nu=2 R / n^{3},
$$

where $R$ is the Rydberg frequency. In a finite interval, $\Delta v$, near the limit there are then $\Delta \nu n^{3} / 2 R$ lines and the integrated intensity is

$$
\int_{\Delta \nu} \frac{J_{n}}{h \nu} d \nu=N_{1} \frac{g_{n}}{g_{1}} \exp .\left(-E_{n} / k T\right) \int_{\Delta \nu} \frac{A_{n} n^{3}}{2 R} d \nu
$$

${ }_{2}$ BS J. Research 10, 771 (1933) RP565.

3 J. Research NBS 17, 45 (1936) RP901. 
where $E_{n}$ is an average value. Since $A_{n} n^{3}$ is a constant for large values of $n$

$$
\int_{\Delta \nu} \frac{J_{n}}{h \nu} d \nu=N_{1} \frac{g_{n}}{g_{1}} \exp .\left(-E_{n} / k T\right) \frac{A_{n} n^{3}}{2 R} \Delta \nu .
$$

The continuous spectrum arises from electrons with a continuous range of kinetic energy, $E$, falling into a level of limiting frequency, $\nu_{i}$, with emission of radiation of frequency, $\nu$, where

$$
h \nu=h \nu_{i}+E \text {. }
$$

The probability of capture into this level can be expressed in terms of the effective collision area, $q$. The number of effective collisions of electrons in an energy range, $\Delta E$, with ions equals the number of quanta in the corresponding frequency range, $\Delta \nu$. In equilibrium the fraction of collisions in the energy range, $\Delta E$, is given by kinetic theory, the number of ions and electrons is equal, and

$$
\frac{J(\nu) \Delta \nu}{h \nu}=\frac{4 \pi^{-1 / 2} h}{\sqrt{2 m}} \frac{N_{e}^{2}}{(k T)^{3 / 2}} \exp .(-E / k T) E q \Delta \nu .
$$

Saha's equation gives for the value of $N_{e}^{2}$

$$
N_{e}^{2}=\frac{(2 \pi m)^{3 / 2}}{h^{3}}(k T)^{3 / 2} \frac{2 g^{+}}{g_{1}} N_{1} \exp .\left(-E_{i} / k T\right),
$$

where $g^{+}$is the weight of the ionized state and $E_{t}$ the ionization energy. Putting this value of $N_{e}^{2}$ in equation 6,

$$
\frac{J(\nu) \Delta \nu}{h \nu}=\frac{16 \pi m}{h^{2}} \frac{g^{+}}{g_{1}} N_{1} \exp \cdot\left(-\frac{E_{t}+E}{k T}\right) E q \Delta \nu .
$$

As $E$ approaches zero, $E q$ remains finite ${ }^{4}$ and the exponent approaches $E_{i} / k T$. In equation 4 the exponent has the same limiting value, and the condition that line and continuous spectra have equal intensities at the limit reduces to

$$
E q=\frac{h^{2}}{32 \pi m R} \frac{g_{n}}{g^{+}} A_{n} n^{3}=1.45 \times 10^{-43} \frac{g_{n}}{g^{+}} A_{n} n^{3} .
$$

This is not a new relation but could be derived from the law that the line absorption equals the continuous absorption at the limit. ${ }^{5}$ The two products, $E_{q}$ and $A n^{3}$, are both roughly proportional to $\lambda$, and this approximation will be used in deriving values at the limit from observed values.

\section{PROBABILITY OF RECOMBINATION INTO 6P OF CESIUM}

Equation 9 will be used to compare experimental values of $A_{n}$ for the subordinate series of cesium and $q$ for the continuous spectrum beyond the $6 \mathrm{P}$ series limit. This is a doublet system with limits $\lambda_{0}=5080$ and 4944, the first being the more intense. For simplicity this will be treated as a single limit at $\lambda_{0}=5080$.

- Mohler and Boeckner, BS J. Research 2, 489 (1929) RP46.

8 Sugiura, J. Phys. 8, 113 (1927).

$106351-36-4$ 
Values ${ }^{6}$ of $A_{n}$ obtained in a limited range of discharge conditions (pressure greater than $60 \mu$ ) in which both $N_{n}$ and $J$ could be evaluated gave

$$
A_{n} n^{3}=5.8 \times 10^{8} \text { at } \lambda 5080
$$

for the sum of the doublet components. The integral on the left side of equation 4 must include all series with the common limit. For the $S$ series, $g_{n} A_{n}$ is 0.1 times the value for the $D$ series and for both

$$
\frac{g_{n}}{g^{+}} A_{n} n^{3}=6.4 \times 10^{9} .
$$

From equation $9, E q=9.3 \times 10^{34}$ at $\lambda=5080$. For comparison with the recombination spectrum, $q$ will be evaluated at $E=0.48 \times 10^{-12} \mathrm{erg}$ or 0.3 electron volt, corresponding to $4520 \mathrm{~A}$.

$$
E q=8.3 \times 10^{-34} . \quad q=1.72 \times 10^{-21} \mathrm{~cm}^{2} .
$$

The experimental value of $q$ based on recombination measurements at low pressures is almost exactly this, viz., $q=1.7 \times 10^{-21}$. The exact agreement is certainly accidental but the precision is sufficient to show that the values of $A$ measured at high pressure are consistent with the low-pressure values of $q$ and not with the high-pressure values which are over 10 times as great. The value $1.7 \times 10^{-21} \mathrm{~cm}^{2}$ is of the same magnitude as the theoretical value for recombination of a 0.3 -volt electron into the $2_{2}$ level of hydrogen, viz., $3.66 \times 10^{-21} \mathrm{~cm}^{2} .^{7}$

These considerations strongly support the conclusion that there is a systematic error in the probe measurements of $N_{e}$ used in computing values of $q$ from the observed intensity of the recombination spectrum. A possible explanation of the error is that the current to the probe lowers the concentration of the electrons in the plasma near the probe. Such an effect would increase with pressure and the magnitude of the effect seems sufficient to account for the discrepancy. The theory of probe measurements is rigorous only at low pressures and the best experimental checks on the theory have been made at low pressures. ${ }^{8}$ There are many possible complications at higher pressures $^{9}$ and no assurance that the electron diffusion effect is predominant.

\section{ELECTRON CONCENTRATION FROM INTENSITY MEASUREMENTS}

There is no reason to doubt the constancy of atomic transition probabilities, and the intensity of the recombination spectrum can be used to compute values of $N_{e}$. The author has published intensity measurements as a function of apparent values of $N_{e}$ for pressures ranging from 0.78 to $290 \mu .^{10}$ There is roughly a twofold change in electron temperature in this range and equation 6 shows that the intensity at the limit varies inversely as $T^{3 / 2}$. Figure 1 gives a

6 J. Research NBS 17, 45 (1936) RP901.

7 Stueckelberg and Morse, Phys. Rev. 36, 16, (1930).

8 Langmuir and Mott-Smith, Gen. Elec. Rev. 27, 449, 538, 616, 762, 810 (1924).

2 Seeliger and Hirchert, Ann. Phys. 5-11, 817 (1931).

10 BS J. Research 10, 771 (1933) RP565. 


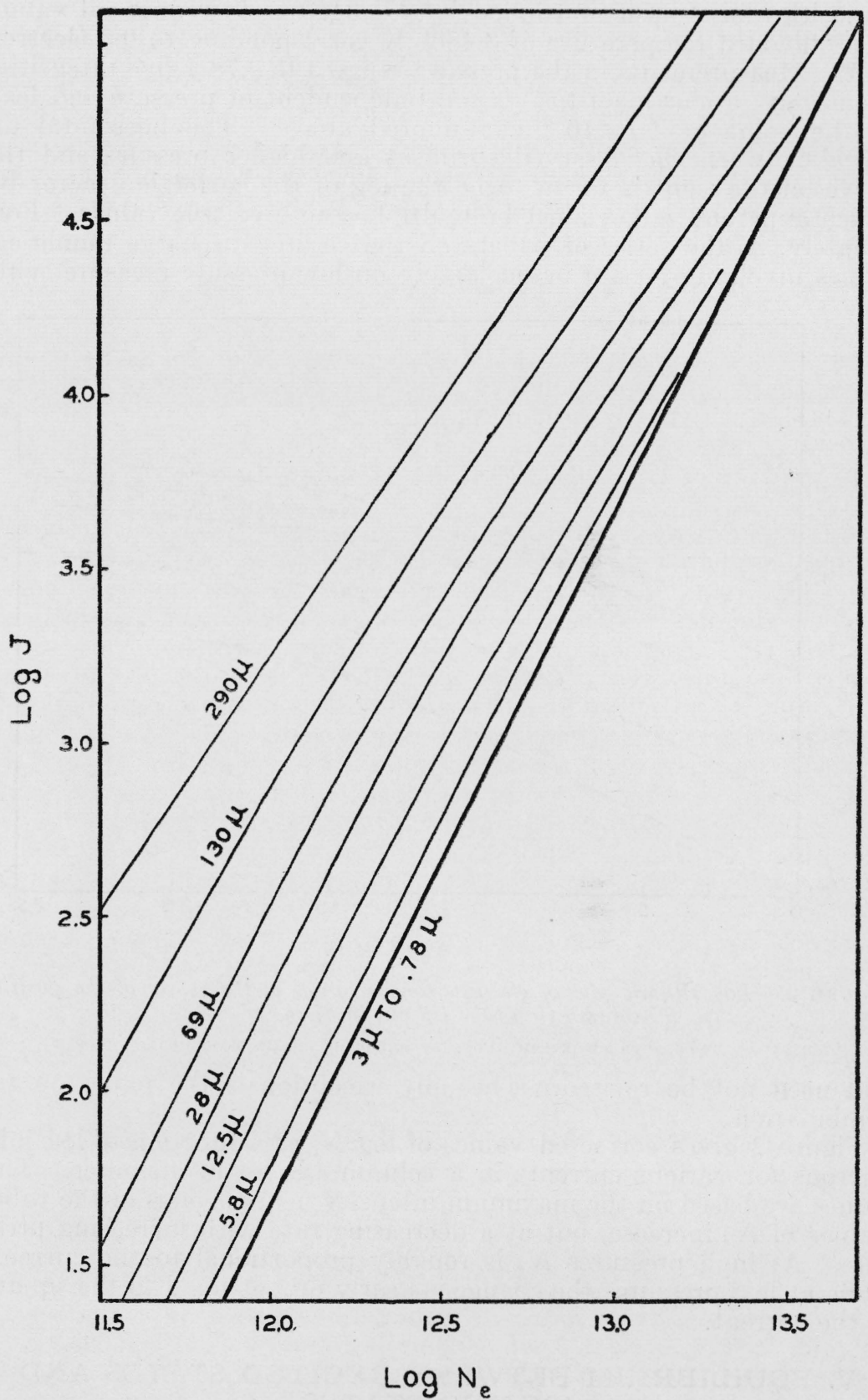

FIGURe 1.-Logarithmic plot of intensity of the continuous spectrum near the $6 \mathrm{P}$ limit versus the observed electron concentration.

Observed values of $\mathrm{J}$ are reduced to a standard electron temperature of $3,480^{\circ} \mathrm{K}$ 
logarithmic plot of intensity versus measured values of $N_{e}$. Observed intensities have been multiplied by $\left(T_{e} / 3480\right)^{3 / 2}$ to reduce all values to a standard temperature of $3,480^{\circ} \mathrm{K}$ corresponding to 0.3 electron volt. Measurements in the pressure range 3 to $0.78 \mu$ give intensities accurately proportional to $N^{2}{ }_{e}$ and independent of pressure and lead to the value $q=1.7 \times 10^{-21} \mathrm{~cm}^{2}$ quoted above. The horizontal interval between the observed curve at any higher pressure and the curve on the right is taken to be the log of the correction factor by which apparent values must be multiplied to give true values. Fortunately, in the series of papers on the cesium discharge numerical values involving $N_{e}$ are based largely on low-pressure measurements

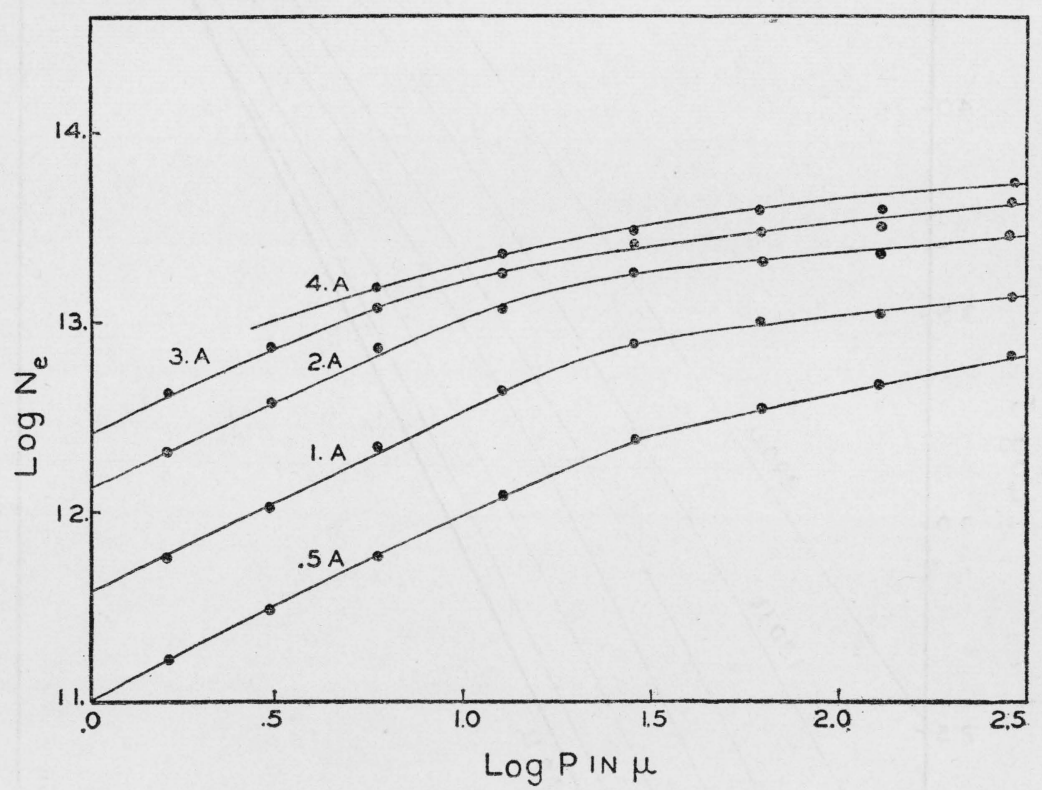

Figure 2.-Logarithmic plot of electron concentration at the center of the positive column in a tube $1.8 \mathrm{~cm}$ in diameter.

Values of $N_{\bullet}$ are based on low-pressure probe measurements and the recombination intensity.

and need not be revised. The only exception is the paper on recombination.

Figure 2 gives corrected values of $\log N_{e}$ as a function of $\log p$ in microns for various currents in a column $1.8 \mathrm{~cm}$ in diameter. The values are based on the maximum intensity near the axis of the tube. Values of $N_{e}$ increase, but at a decreasing rate with increasing pressure. At high pressures $N_{e}$ is roughly proportional to the current, while at low pressures the change is nearly proportional to the square of the current.

\section{EQUILIBRIUM BETWEEN EXCITED STATES AND IONIZED STATES}

Direct measurements of the reversal temperature of the higher series lines of the principal series ${ }^{11}$ and intensity measurements in $\mathrm{S}, \mathrm{D}$, and $\mathrm{F}$ series indicate that at pressures of the order of $100 \mu$ all

\footnotetext{
11 J. Research NBS 16, 227 (1936) RP859; 1\%, 45 (1936) RP901.
} 
states beyond the third in each series have the same reversal temperature. The number of atoms in a given state, $N_{n}$, is related to the reversal temperature, $T_{n}$, by an equation of the Boltzman form.

$$
N_{n}=N_{1} \frac{g_{n}}{g_{1}} \exp .\left(-E_{n} / k T_{n}\right) .
$$

There is then a temperature distribution of excitation among the higher states and the theoretical considerations of the first section suggest that this common value of $T_{n}$ must be related to the electron concentration, $N_{e}$. By equation 10 a value of $T_{n}$ is defined by a given value, $N_{n}$, without any assumption as to equilibrium, and in the same way one can define a temperature, $T_{s}$, by the use of Saha's equation and a given value of $N_{e}$,

$$
N_{e}^{2}=2.43 \times 10^{15} \frac{g^{+}}{g_{1}} N_{1} T_{s}^{3 / 2} \exp .\left(-E_{i} / k T_{s}\right)
$$

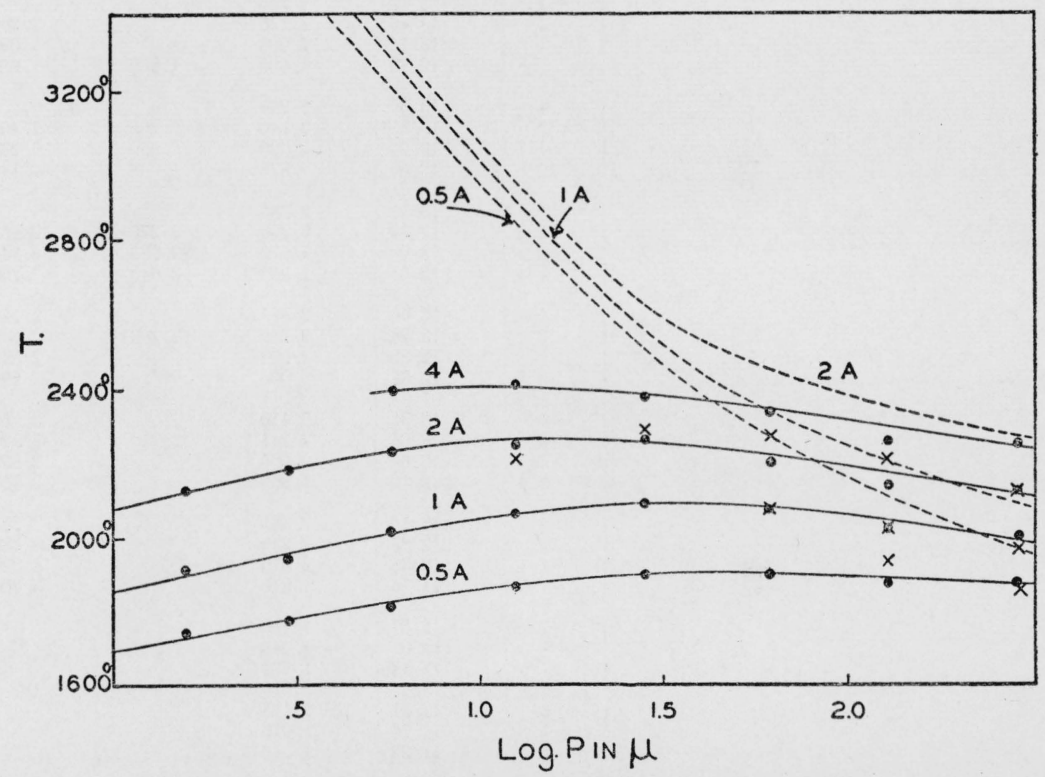

FIgURE 3.-Full lines and dots give $T_{s}$ based on values of $N_{e}$ from figure 2 and Saha's equation; crosses give reversal temperatures of higher series lines and broken lines give the reversal temperatures of the resonance doublet which is nearly equal to $T_{e}$.

It can be seen from the derivation of equation 9 that if the line intensity approaches the continuous intensity at the limit then $T_{n}$ must approach $T_{s}$.

Values of $N_{e}$ from figure 2 have been used to compute values of $T_{s}$, and table 1 and figure 3 show that there is a fairly satisfactory agreement between $T_{n}$ and $T_{s}$ in the range in which $T_{n}$ can be evaluated. This consistency of the results justifies the method of basing values of $N_{e}$ on intensity measurements, for the correction brings a wide range of experimental data into fair agreement.

The common value of $T_{n}$ and $T_{s}$ is much less than the electron temperature, while the reversal temperature of the first doublet of the principal series, $T(2 \mathrm{P})$, is nearly equal to the electron temperature 
except at very low pressures. ${ }^{12}$ Values of $T(2 \mathrm{P})$ have been included in figure 3 and table 1 . The two temperatures, $T_{e}$ and $T_{s}$, define the electrical conditions in the plasma. The one gives the velocity distribution of the electrons and the other the number of electrons and ions per cubic centimeter for a given value of $N_{a}$. At high pressures where electrical methods fail, both can be measured by optical methods. The intensity of the continuum near a series limit gives $T_{s}$, and the intensity distribution depends on $T_{e}{ }^{13}$

TABLE 1.-Electron concentration, $T_{s}$, and reversal temperatures for a positive column in cesium vapor

\begin{tabular}{|c|c|c|c|c|c|}
\hline$P$ & Current & $\begin{array}{c}\text { Log } N_{i} \\
\text { corrected }\end{array}$ & $T s$ & $\begin{array}{l}T_{n} \text { higher } \\
\text { lines }\end{array}$ & $T(6 \mathrm{P})$ \\
\hline${ }^{\mu}$ & $\begin{array}{r}a m p \\
4 \\
2 \\
1 \\
\quad .5\end{array}$ & $\begin{array}{l}13.73 \\
13.46 \\
13.14 \\
12.82\end{array}$ & $\begin{array}{l}{ }^{\circ} \mathrm{K} \\
2,250 \\
2,125 \\
2,000 \\
1,880\end{array}$ & $\begin{array}{l}2,115 \\
1,970 \\
1,860\end{array}$ & $\begin{array}{r}2,260 \\
2,080 \\
1,950\end{array}$ \\
\hline $130 \ldots$ & $\begin{array}{l}4 \\
2 \\
1 \\
.5\end{array}$ & $\begin{array}{l}13.59 \\
13.35 \\
13.04 \\
12.66\end{array}$ & $\begin{array}{l}2,260 \\
2,140 \\
2,020 \\
1,875\end{array}$ & $\begin{array}{l}2,205 \\
2,030 \\
1,938\end{array}$ & $\begin{array}{r}2,400 \\
2,230 \\
2,140\end{array}$ \\
\hline $69 \ldots$ & $\begin{array}{l}4 \\
2 \\
1 \\
.5\end{array}$ & $\begin{array}{l}13.58 \\
13.32 \\
13.00 \\
12.55\end{array}$ & $\begin{array}{l}2,335 \\
2,200 \\
2,070 \\
1,900\end{array}$ & $\begin{array}{l}2,270 \\
2,080 \\
2,045\end{array}$ & $\begin{array}{r}2,450 \\
2,340 \\
2,260\end{array}$ \\
\hline $28 \ldots$ & $\begin{array}{l}4 \\
2 \\
1 \\
.5\end{array}$ & $\begin{array}{l}13.46 \\
13.26 \\
12.88 \\
12.38\end{array}$ & $\begin{array}{l}2,375 \\
2,200 \\
2,090 \\
1,900\end{array}$ & $(2,290)$ & $\begin{array}{l}2,740 \\
2,600 \\
2,530 \\
2,500\end{array}$ \\
\hline $12.5 \ldots$ & $\begin{array}{l}\stackrel{4}{2} \\
1 \\
.5\end{array}$ & $\begin{array}{l}13.36 \\
13.05 \\
12.64 \\
12.10\end{array}$ & $\begin{array}{l}2,410 \\
2,245 \\
2,060 \\
1,860\end{array}$ & $(2,210)$ & $\begin{array}{l}3,050 \\
2,920 \\
2,890 \\
2,860\end{array}$ \\
\hline $5,8 \ldots \ldots$ & $\begin{array}{l}4 \\
2 \\
1 \\
\quad .5\end{array}$ & $\begin{array}{l}13.15 \\
12.85 \\
12.33 \\
11.76\end{array}$ & $\begin{array}{l}2,385 \\
2,230 \\
2,010 \\
1,810\end{array}$ & - & $\begin{array}{l}3,280 \\
3,280 \\
3,200\end{array}$ \\
\hline $3 \ldots$ & $\begin{array}{l}2 \\
1 \\
.5\end{array}$ & $\begin{array}{l}12.57 \\
12.02 \\
11.48\end{array}$ & $\begin{array}{l}2,180 \\
1,940 \\
1,775\end{array}$ & & - \\
\hline $1.6 \ldots$ & $\begin{array}{l}2 \\
1 \\
.5\end{array}$ & $\begin{array}{l}12.31 \\
11.76 \\
11.22\end{array}$ & $\begin{array}{l}2,125 \\
1,910 \\
1,740\end{array}$ & & $\cdots$ \\
\hline
\end{tabular}

The elementary interactions between electrons, atoms, and radiation in a discharge become very complicated with increasing pressure, but these processes gradually approach equilibrium with each other so that statistically the discharge conditions become simpler as pressure and current increase. At extremely low pressures the plasma electrons take up a temperature distribution of velocity. (The mechanism is not entirely clear.) Above a pressure of $10 \mu$ collisions of the first and second kind keep the number of atoms in the first excited state nearly in equilibrium with the electron temperature. Ionization of highly excited states and recombination into these states bring the number of ions and of excited states to equilibrium near $60 \mu$. Above this pressure the intensity of the higher series lines and of the continuum near the limit is characteristic of the temperature, $T_{s}$, but the population of the first excited state and the intensity distri-

12 BS J. Research 9, 493 (1932) RP485.

13 Mohier and Boeckner, BS J. Research 3, 303 (1929) RP96. 
bution in the continuum depend on $T_{e}$. It is seen from figure 3 that these two temperatures approach each other with increasing pressure. Presumably recombination on the tube walls keeps $T_{s}$ below $T_{e}$, and they will approach equality when spacere combination becomes predominant. Then one temperature will define electrical conditions and spectral intensity distribution for a given value of $N_{a}$. The gas temperature has been assumed to be the wall temperature, but is probably appreciably above this at the higher pressures, and the value of $N_{a}$ becomes uncertain.

Studies of ares at atmospheric pressure ${ }^{14}$ indicate that the gas temperature in the core of the discharge is nearly equal to the electron temperature. This last stage in the approach to equilibrium is probably not approached in the range of pressure studied here.

I am indebted to Dr. C. Boeckner for pointing out the possibility that probe measurements of electron concentration may be systematically low at higher pressures.

Washington, October 16, 1936.

14 Mannkopfi, Z. Phys. 86, 161 (1933). 